\title{
Numerical solution of the two-phase tumour growth model with moving boundary
}

\author{
Gopikrishnan C. Remesan ${ }^{1}$
}

(Received 23 January 2019; revised 16 May 2019)

\begin{abstract}
A novel numerical technique is proposed to solve a two-phase tumour growth model in one spatial dimension without needing to account for the boundary dynamics explicitly. The equivalence to the standard definition of a weak solution is proved. The method is tested against equations with analytically known solutions, to illustrate the advantages over existing techniques. The tumour growth model is solved using the new procedure and is shown to be consistent with results available in the literature.
\end{abstract}

\section{Contents}

\section{Introduction}

DOI:10.21914/anziamj.v60i0.13936 gives this article, (c) Austral. Mathematical Soc. 2019. Published June 15, 2019, as part of the Proceedings of the 18th Biennial Computational Techniques and Applications Conference. ISSN 1445-8810. (Print two pages per sheet of paper.) Copies of this article must not be made otherwise available on the internet; instead link directly to the DOI for this article. 


\section{Extended model}

3 Numerical experiments

3.1 Case $1 \ldots \ldots \ldots \ldots \ldots \ldots$

3.2 Case 2

\section{Introduction}

We consider the tumour growth model presented in the seminal paper by Breward et al. [2]. The partial differential equations are defined in a timedependent one-dimensional spatial domain. Such systems generally account for higher spatial dimensional models reduced to a single spatial dimension by symmetry arguments [3]. In the current model, tumour cells and the surrounding fluid medium are considered as two distinct, actively interacting phases. The cell phase is viscous with viscosity $\mu$, and the fluid phase is inviscid.

Let $\Omega(t)=(0, \ell(t))$ be the interval representing the tumour where $\ell(t)$ is the tumour radius at time $t$. Define the space-time domain of the tumour $\mathrm{D}_{\mathrm{T}}:=\cup_{0<\mathrm{t}<\mathrm{T}}\{\mathrm{t}\} \times \Omega(\mathrm{t})$ and the time-dependent boundary $\mathrm{B}_{\mathrm{T}}:=$ $\partial D_{T} \backslash\left(\{T\} \times \Omega(T) \cup\{0\} \times \Omega(0) \cup[0, T] \times\{0\}\right.$ ) (Figure 1 ). The surface $B_{T}$ is the space-time bounding surface of tumour, except for the top, bottom and left boundaries which are time-independent, and $\mathrm{B}_{\mathrm{T}}$ is assumed to be of class $\mathrm{e}^{1}$ [4, p. 627].

The model seeks the variables $\alpha, \mathfrak{u}_{c}$ and $C$ that denote volume fraction of the tumour cells, velocity of the tumour cells and oxygen tension, respectively, 


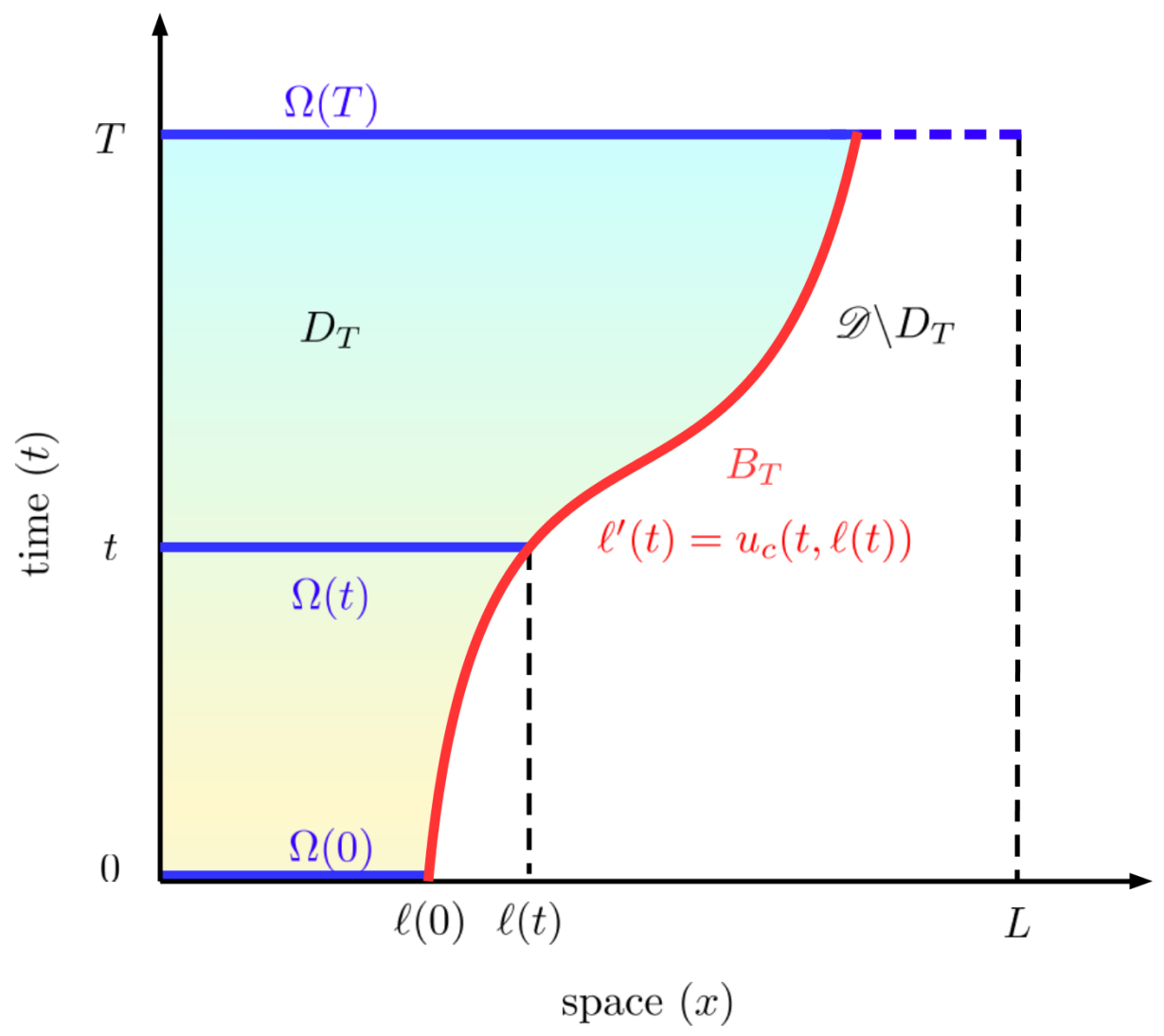

Figure 1: The rectangle $(0, T) \times(0, L)$ is the time-independent domain $\mathcal{D}$. The region to the left of the red curve $B_{T}$ is the domain of the tumour $D_{T}$ and to the right of curve $B_{T}$ is $\mathcal{D} \backslash D_{T}$. 
such that the following hold in $\mathrm{D}_{\mathrm{T}}$ :

$$
\begin{aligned}
\frac{\partial \alpha}{\partial t}+\frac{\partial}{\partial x}\left(u_{c} \alpha\right) & =\alpha f(\alpha, C) \\
\frac{k u_{c} \alpha}{1-\alpha}-\mu \frac{\partial}{\partial x}\left(\alpha \frac{\partial u_{c}}{\partial x}\right) & =-\frac{\partial}{\partial x}\left[\alpha \frac{\alpha-\alpha^{*}}{(1-\alpha)^{2}} H\left(\alpha-\alpha_{\min }\right)\right] \\
\frac{\partial C}{\partial t}-\frac{\partial^{2} C}{\partial x^{2}} & =-\frac{Q \alpha C}{1+\hat{Q}_{1} C}
\end{aligned}
$$

where

$$
f(\alpha, C)=\frac{\left(1+s_{1}\right)(1-\alpha) C}{1+s_{1} C}-\frac{s_{2}+s_{3} C}{1+s_{4} C} .
$$

The positive, constant drag coefficient $k$ controls the drag between the phases. The constants $\alpha^{*}$ and $\alpha_{\min }$ are positive and control the stress in the cell phase. The positive constant $s_{1}$ controls the cell birth rate, while the positive constants $s_{2}, s_{3}$ and $s_{4}$ control the cell death rate. The Heaviside function $H(x)=1$ if $x \geqslant 0$ and zero otherwise. The non-negative constants $Q$ and $\hat{Q}_{1}$ control the oxygen consumption rate of the tumour cells. The two-phase model of Breward et al. [2] uses a quasi-steady state assumption for oxygen tension which is relaxed in this study. This means the explicit temporal variation of oxygen tension is considered which makes (1c) a parabolic equation.

The initial and boundary conditions are

$$
\begin{aligned}
& \alpha(0, x)=\alpha_{0}(x) \text { and } C(0, x)=C_{0}(x) \text { for all } x \in \Omega(0), \\
& u_{c}(t, 0)=0, \quad \mu \frac{\partial u_{c}(t, \ell(t))}{\partial x}=\frac{\alpha(t, \ell(t))-\alpha_{\min }}{[1-\alpha(t, \ell(t))]^{2}} H\left[\alpha(t, \ell(t))-\alpha_{\min }\right]
\end{aligned}
$$

$\frac{\partial C(t, 0)}{\partial x}=0$ and $C(t, \ell(t))=1$ for all $t \in(0, T)$,

$\ell^{\prime}(t)=u_{c}(t, \ell(t))$ for all $t, \quad \ell(0)=\ell_{0}$.

Here, we assume that $\alpha_{0}(x)$ satisfies $0<m_{\alpha} \leqslant \alpha_{0}(x) \leqslant M_{\alpha}<1$ for every $x \in \Omega(0)$ where $m_{\alpha}$ and $M_{\alpha}$ are constants. 
The standard method for solving the system (1a)-(1g) is to transform the domain $\Omega(t)$ into a fixed interval using a suitable change of variables [1, 7,8]. An inverse transform is then applied to obtain the solution in the moving domain. Even though this method is commonly adopted, it comes with significant drawbacks. Firstly, the change of variable is computable only when the geometry of the problem is simple enough. This is even harder in 2D and 3D domains. Secondly, for the clear choice of $x \rightarrow \xi:=x / \ell(t)$ in the $1 \mathrm{D}$ case, the discretisation error is proportional to $\ell(t) \Delta \xi$. An alternative choice is to discretise $(0, \ell(t))$ and apply a numerical scheme. In this method re-meshing is required at each time step, which may become computationally expensive.

This article introduces a new numerical technique that overcomes theses disadvantages. This novel method, referred to as extended model, presents solutions on a larger domain that contains all the time-dependent domains $\Omega(t)$ for a finite time. This domain, referred to as the extended domain, is timeindependent and requires only one initial spatial discretisation, thereby avoiding the need to re-mesh. Also, the discretisation error becomes independent of $\ell(t)$.

Section 2 introduces the extended model and proves its equivalence to the standard model. Section 3 applies the new numerical technique to a simplified version of extended model and the results are compared with the known exact solution. The effect of parameters in the new method is also investigated. The extended model is solved using the numerical technique developed and compared with results available from the literature.

\section{$2 \quad$ Extended model}

This section introduces weak solutions in both the given domain and the extended domain. The solutions for the extended and original domains are proved to be equivalent. For $p \in[1, \infty]$ and a family of domains $\{\Omega(t)\}_{0 \leqslant t \leqslant T}$, 
define

$$
\begin{aligned}
\mathbb{L}^{\mathrm{p}}\left(0, \mathrm{~T} ; \mathrm{H}^{1}(\Omega(\mathrm{t}))\right):= & \left\{v:[0, \mathrm{~T}] \times \mathbb{R} \rightarrow \mathbb{R} \mid v(\cdot, \mathrm{t}) \in \mathrm{H}^{1}(\Omega(\mathrm{t})) ;\right. \\
& \left.\forall \mathrm{t} \in[0, \mathrm{~T}], \quad\|\| v(\cdot, \mathrm{t})\left\|_{\mathrm{H}^{1}}\right\|_{\mathrm{L}^{\mathrm{p}}(0, \mathrm{~T})}<\infty\right\} .
\end{aligned}
$$

Multiply (1a) by a test function $\phi \in \mathcal{C}_{c}^{\infty}\left(\bar{D}_{\mathrm{T}} \backslash[\{\mathrm{T}\} \times \Omega(\mathrm{T})]\right)$ and apply integration by parts. With $\nabla_{t, x}:=\left(\partial_{t}, \partial_{x}\right)$, applying (1g) and (1d) yields

$$
\int_{D_{\mathrm{T}}}\left[\alpha f(\alpha, C) \phi+\left(\alpha, u_{c} \alpha\right) \cdot \nabla_{t, x} \phi\right] d t d x+\int_{\Omega(0)} \phi(0, x) \alpha_{0}(x) d x=0 .
$$

This constitutes the weak formulation of the hyperbolic conservation law. The weak solutions in the extended and original domains are defined below. Firstly, we give the definition of the solution in the domain $\mathrm{D}_{\mathrm{T}}$.

Definition 1 (weak solution I). A weak solution of the system (1) in $\mathrm{D}_{\mathrm{T}}$ is a four-tuple $\left(\alpha, \mathrm{u}_{\mathrm{c}}, \mathrm{C}, \Omega\right)$ such that $0<\overline{\mathrm{m}}_{\alpha} \leqslant \alpha \leqslant \bar{M}_{\alpha}<1$ where $\overline{\mathrm{m}}_{\alpha} \leqslant \mathrm{m}_{\alpha}$ and $\mathrm{M}_{\alpha} \leqslant \bar{M}_{\alpha}$ are time-independent constants, $\mathrm{C} \geqslant 0$ and

1. $\alpha \in \mathrm{L}^{\infty}\left(\mathrm{D}_{\mathrm{T}}\right)$ satisfies (2) for every $\phi \in \mathcal{C}_{\mathrm{c}}^{\infty}\left(\overline{\mathrm{D}}_{\mathrm{T}} \backslash[\{\mathrm{T}\} \times \Omega(\mathrm{T})]\right)$;

2. $\mathrm{u}_{\mathrm{c}} \in \mathbb{L}^{\infty}\left(0, \mathrm{~T} ; \mathrm{H}^{1}(\Omega(\mathrm{t}))\right)$ with $\left.\mathrm{u}_{\mathrm{c}}\right|_{\mathrm{x}=0}=0$ and $\mathrm{C} \in \mathbb{L}^{2}\left(0, \mathrm{~T} ; \mathrm{H}^{1}(\Omega(\mathrm{t}))\right)$ with $\left.C\right|_{x=\ell(\mathrm{t})}=1$ are solutions of $(1 \mathrm{~b})$ and $(1 \mathrm{c})$ in the sense of distributions;

3. the domain $\Omega(\mathrm{t})$ is the open interval $(0, \ell(\mathrm{t}))$ where $\ell(\mathrm{t})$ is governed by $(1 \mathrm{~g})$.

Secondly, we give the definition of the weak solution in the extended domain $\mathcal{D}=(0, T) \times(0, L)$ (Figure 1 ) where $L$ is chosen such that $\ell(t)<L$ for every $t \leqslant T$. 
Definition 2 (weak solution II). A weak solution of the system (1) in the extended domain $\mathcal{D}$ (Figure 1) is a four-tuple $\left(\tilde{\alpha}, \tilde{u}_{\mathfrak{c}}, \tilde{\mathrm{C}}, \tilde{\Omega}\right)$ such that $0<$ $\overline{\mathrm{m}}_{\alpha} \leqslant\left.\tilde{\alpha}\right|_{\tilde{\Omega}} \leqslant \bar{M}_{\alpha}<1, \tilde{\mathrm{C}} \geqslant 0$ and

1. $\tilde{\alpha} \in \mathrm{L}^{\infty}(\mathcal{D})$ such that for every $\tilde{\phi} \in \mathcal{C}_{\mathcal{c}}^{\infty}([0, T) \times(0, L))$ :

$$
\int_{\mathcal{D}}\left[\tilde{\alpha} f(\tilde{\alpha}, \tilde{C}) \tilde{\phi}+\left(\tilde{\alpha}, \tilde{u}_{c} \tilde{\alpha}\right) \cdot \nabla_{t, x} \tilde{\phi}\right] d t d x+\int_{\Omega(0)} \tilde{\phi}(0, x) \alpha_{0}(x) d x=0 ;
$$

2. for a fixed $\mathrm{t}, \tilde{\Omega}(\mathrm{t}):=\{x: \tilde{\alpha}(\mathrm{t}, \mathrm{x})>0\}, \tilde{\mathrm{u}}_{\mathrm{c}}=0, \tilde{\mathrm{C}}=1$ on $(0, \mathrm{~L}) \backslash \tilde{\Omega}(\mathrm{t})$. Define $\tilde{\mathrm{D}}_{\mathrm{T}}:=\cup_{0<\mathrm{t}<\mathrm{T}}\{\mathrm{t}\} \times \tilde{\Omega}(\mathrm{t})$;

3. $\tilde{\mathfrak{u}}_{\mathrm{c}} \in \mathrm{L}^{\infty}(\mathcal{D})$ with $\left.\mathfrak{u}_{\mathrm{c}}:=\left.\tilde{\mathfrak{u}}_{\mathrm{c}}\right|_{\tilde{D}_{\mathrm{T}}}, \mathfrak{u}_{\mathrm{c}} \in \mathbb{L}^{\infty}\left(0, \mathrm{~T} ; \mathrm{H}^{1}(\tilde{\Omega}(\mathrm{t}))\right)\right)$ and, $\tilde{\mathrm{C}} \in$ $\mathrm{L}^{2}(\mathcal{D})$ such that $\mathrm{C}:=\left.\tilde{\mathrm{C}}\right|_{\tilde{\mathrm{D}}_{\mathrm{T}}} \in \mathbb{L}^{2}\left(0, \mathrm{~T} ; \mathrm{H}^{1}(\tilde{\Omega}(\mathrm{t}))\right)$ are solutions of $(1 \mathrm{~b})$ and (1c) in the sense of distributions.

Theorem 3. If $\left(\alpha, \mathfrak{u}_{\mathfrak{c}}, \mathrm{C}, \Omega\right)$ is a weak solution I, then $\left(\tilde{\alpha}, \tilde{u}_{\mathfrak{c}}, \tilde{\mathrm{C}}, \tilde{\Omega}\right)$ defined by $\tilde{\alpha}:=\alpha, \tilde{u}_{c}:=u_{c}$ and $\tilde{C}:=C$ in $\mathrm{D}_{\mathrm{T}}$ and, $\tilde{\alpha}:=0, \tilde{u}_{\mathrm{c}}:=0, \tilde{\mathrm{C}}:=1$ in $\mathcal{D} \backslash \mathrm{D}_{\mathrm{T}}$ with $\tilde{\Omega}(\mathrm{t}):=\Omega(\mathrm{t})$ is a weak solution II. Conversely, if $\left(\tilde{\alpha}, \tilde{u}_{\mathfrak{c}}, \tilde{\mathrm{C}}, \tilde{\Omega}\right)$ is a weak solution II, then $\left(\alpha, \mathfrak{u}_{\mathfrak{c}}, C, \Omega\right)$ with $\Omega=\tilde{\Omega}$ and $\alpha:=\left.\tilde{\alpha}\right|_{\tilde{D}_{\mathrm{T}}}, \mathfrak{u}_{\mathrm{c}}:=\left.\tilde{\mathrm{u}}_{\mathrm{c}}\right|_{\tilde{\mathrm{D}}_{\mathrm{T}}}$ and $\mathrm{C}:=\left.\tilde{\mathrm{C}}\right|_{\tilde{\mathrm{D}}_{\mathrm{T}}}$ is a weak solution $I$.

Proof: Let $\left(\alpha, u_{c}, C, \Omega\right)$ be a weak solution I and $\tilde{\phi} \in \mathcal{C}_{c}^{\infty}([0, T) \times(0, L))$. Since $\left.\tilde{\phi}\right|_{\mathrm{D}_{\mathrm{T}}} \in \mathcal{C}_{\mathrm{c}}^{\infty}\left(\overline{\mathrm{D}}_{\mathrm{T}} \backslash(\{\mathrm{T}\} \times \Omega(\mathrm{T}))\right)$, (2) holds true. Let $\tilde{\alpha}=\alpha$ in $\mathrm{D}_{\mathrm{T}}$ and $\tilde{\alpha}=0$ in $\mathcal{D} \backslash D_{\mathrm{T}}$. Then applying the definitions of $\tilde{u}_{c}$ and $\tilde{C}$ from the statement of Theorem 3 yields

$$
\begin{array}{r}
\int_{D_{T}}\left(\tilde{\alpha} f(\tilde{\alpha}, \tilde{C}) \tilde{\phi}+\left(\tilde{\alpha}, \tilde{u}_{c} \tilde{\alpha}\right) \cdot \nabla_{t, x} \tilde{\phi}\right) d t d x+\int_{\Omega(0)} \tilde{\phi}(0, x) \alpha_{0}(x) d x=0, \\
\int_{\mathcal{D} \backslash D_{T}}\left(\tilde{\alpha}, \tilde{u}_{c} \tilde{\alpha}\right) \cdot \nabla_{t, x} \tilde{\phi} d t d x+\int_{{\mathcal{D} \backslash D_{T}}} \tilde{\alpha} f(\tilde{\alpha}, \tilde{C}) \tilde{\phi} d t d x=0 .
\end{array}
$$


Add (4) and (5) to obtain

$$
\int_{\mathcal{D}}\left(\tilde{\alpha} f(\tilde{\alpha}, \tilde{C}) \tilde{\phi}+\left(\tilde{\alpha}, \tilde{u}_{c} \tilde{\alpha}\right) \cdot \nabla_{t, x} \tilde{\phi}\right) d t d x+\int_{\Omega(0)} \tilde{\phi}(0, x) \alpha_{0}(x) d x=0 .
$$

Therefore (3) holds true. The conditions on $\tilde{u}_{c}$ and $\tilde{C}$ follow naturally from Definition 2. Since $\tilde{\alpha}>0$ in $D_{\mathrm{T}}$ and $\tilde{\alpha}=0$ in $\mathcal{D} \backslash D_{\mathrm{T}}, \tilde{\Omega}(\mathrm{t})=\Omega(\mathrm{t})$ for every $\mathrm{t} \in[0, \mathrm{~T})$. Therefore $\left(\tilde{\alpha}, \tilde{\mathrm{u}}_{\mathrm{c}}, \tilde{\mathrm{C}}, \tilde{\Omega}\right)$ is a weak solution II.

Conversely, assume that $\left(\tilde{\alpha}, \tilde{u}_{c}, \tilde{C}, \tilde{\Omega}\right)$ is a weak solution II and let $\phi \in$ $\mathcal{C}_{c}^{\infty}\left(\bar{D}_{\mathrm{T}} \backslash(\{\mathrm{T}\} \times \Omega(\mathrm{T}))\right)$. Define $\tilde{\phi} \in \mathcal{C}_{\mathrm{c}}^{\infty}([0, \mathrm{~T}) \times(\mathrm{O}, \mathrm{L}))$ such that $\tilde{\phi}=\phi$ in $D_{\mathrm{T}}$. Since $\Omega(t)=\Omega(t)$ for every $t, \tilde{\alpha}=0$ in $\mathcal{D} \backslash D_{\mathrm{T}}$, and using this in (3) we obtain (2). We now recover $(1 \mathrm{~g})$. For this, define a vector field $\mathbf{F}: \mathcal{D} \rightarrow \mathbb{R}^{2}$ by $\mathbf{F}(t, x):=\left(\tilde{\alpha}, \tilde{u}_{c} \tilde{\alpha}\right)$. We set $\left.\mathbf{F}\right|_{\mathrm{B}_{\mathrm{T}}^{+}}=\left.\left.\mathbf{F}\right|_{\mathrm{D}_{\mathrm{T}}}\right|_{\mathrm{B}_{\mathrm{T}}}$ and $\left.\mathbf{F}\right|_{\mathrm{B}_{\bar{\tau}}^{-}}=\left.\left.\mathbf{F}\right|_{\mathcal{D} \backslash \mathrm{D}_{\mathrm{T}}}\right|_{\mathrm{B}_{\mathrm{T}}}$. Since the weak divergence of the vector field $\mathbf{F}$ is $-\tilde{\alpha} f(\tilde{\alpha}, \tilde{C}) \in L^{2}(\mathcal{D})$, the flux of $\mathbf{F}$ is continuous across $B_{T}$. Since $\tilde{\alpha}=0$ in $\mathcal{D} \backslash D_{\mathrm{T}},\left.\mathbf{F}\right|_{B_{\mathrm{T}}^{-}}=\mathbf{0}$. Therefore, $\left(\left.\mathbf{F}\right|_{\mathrm{B}_{\mathrm{T}}^{+}}-\left.\mathbf{F}\right|_{\mathrm{B}_{\mathrm{T}}^{-}}\right) \cdot \mathbf{n}_{\mathrm{B}_{\mathrm{T}}}=\left(\alpha, \mathrm{u}_{\mathrm{c}} \alpha\right) \cdot \mathbf{n}_{\mathrm{B}_{\mathrm{T}}}=0$ where $\mathbf{n}_{\mathrm{B}_{\mathrm{T}}}$ is the normal to $\mathrm{B}_{\mathrm{T}}$ given by $\left(\left|\ell^{\prime}(t)\right|^{2}+1\right)^{-1 / 2}\left(-\ell^{\prime}(t), 1\right)$. This gives $\left(\alpha, u_{c} \alpha\right) \cdot \mathbf{n}_{B_{T}}=0$. Since $\alpha>0$, $\ell^{\prime}(t)=u_{c}(t, \ell(t))$. The conditions on $\mathfrak{u}_{c}$ and $C$ follows directly from the definitions. Therefore $\left(\alpha, \mathfrak{u}_{\mathfrak{c}}, \mathrm{C}, \Omega\right)$ is a weak solution I.

This completes the proof of the equivalence between the solutions.

\section{Numerical experiments}

By Theorem 3, solving (1a) in the extended domain $(0, \mathrm{~L})$ and then taking its restriction, as in the statement of Theorem 3, gives the solution in the domain $\mathrm{D}_{\mathrm{T}}$. Equation (1a) is solved using cell-centred finite volume methods. In particular, we use two methods to solve the volume fraction equation: upwinding with Godunov flux [5, p. 135] (method U), and (monotonic upwind 
scheme for conservation laws) with Godunov flux [5, p. 146] (method M). The uniform space and time discretisations are $0=x_{0}<x_{1}<\cdots<x_{i}<$ $\cdots<x_{M}=L, 0=t_{0}<t_{2}<\cdots<t_{j}<\cdots<t_{N}=T$ with $h=x_{i+1}-x_{i}$ and $\Delta t=t_{j+1}-t_{j}$. The right hand side boundary $\ell(t)$ is approximated by $\ell_{h}(t)=\min _{x}\left\{x: \tilde{\alpha}<\alpha_{\text {thr }}\right.$ on $\left.(x, L)\right\}$ where threshold $\alpha_{\text {thr }}$ is a small positive number. Define $\tilde{\alpha}:=0$ for $x \geqslant \ell_{h}(t)$ to eliminate the error caused by small positive values of $\tilde{\alpha}$ (created by numerical diffusion). Equations (1b) and (1c) are solved using a conforming $\mathrm{P}_{1}$ finite element method (FEM) in space and forward finite difference in time, in the reconstructed domain $\left(0, \ell_{h}(t)\right)$. This procedure, named scheme A, is outlined below.

1. Start at $t_{0}=0$. Solve $\tilde{u}_{c, h}^{0}$ using $\tilde{\alpha}_{h}^{0}$ (initial condition).

For $j=1$ to $N, t_{j}=t_{j-1}+\Delta t$ :

2. $\ell_{h}^{j}=\min _{x_{i}}\left\{x_{i}: \tilde{\alpha}_{h}^{j-1}<\alpha_{\text {thr }}\right.$ on $\left.\left(x_{i}, L\right)\right\}$;

3. find $\tilde{u}_{c, h}^{j}$ and $\tilde{C}_{h}^{j}$ in $\left(0, \ell_{h}^{j}\right)\left(P_{1}\right.$ conforming FEM);

4. extrapolate $\tilde{u}_{c, h}^{j}=0$ and $\tilde{C}_{h}^{j}=1$ to $\left(\ell_{h}^{j}, L\right)$;

5. find $\tilde{\alpha}_{h}^{j}$ in $(0, L)$ (using method $U$ or $M$ ).

The major advantages of scheme A are the complete elimination of re-meshing and applicability in higher dimensions. Scheme B denotes the procedure of obtaining a numerical solution in the scaled domain $(0,1)$ [2]. Two test cases are considered in the numerical experiments.

In the first test case (Section 3.1), the cell velocity $\mathfrak{u}_{\mathfrak{c}}$ and the oxygen tension $\mathrm{C}$ are assumed to be unity. In this case (1a) reduces to a semilinear advection equation which is solved analytically by the method of characteristics. Figure 2 compares the analytical solution with the numerical solutions. We also study the influence of $\alpha_{\text {thr }}$ on locating the tumour frontier. In the second case (Section 3.2), we compare the approximate solutions of the full system (1a)-(1g). 
In all numerical tests, to preserve conformity with Breward et al. [2], the parameters are set to $s_{1}=10=s_{4}, s_{2}=0.5=s_{3}, k=1=\mu, Q=0.5$, $\widehat{\mathrm{Q}}_{1}=0$ and $\ell(0)=1$.

\subsection{Case 1}

The analytical solution to (1a) in the case where $C=\mathfrak{u}_{c}=1$ is

$$
\alpha(t, x)=\frac{\left(c_{2}-c_{1}\right) \alpha_{0}(x-t) \exp \left[\left(c_{1}-c_{2}\right) t\right]}{c_{1} \alpha_{0}(x-t]\left(1-\exp \left[\left(c_{1}-c_{2}\right) t\right]\right)+c_{2}-c_{1}},
$$

where $c_{1}=1$ and $c_{2}=\left(s_{2}+s_{3}\right) /\left(1+s_{4}\right)$. The tested initial data $\alpha(0, t)=$ $\alpha_{0}(x)$ are:

(i) $\alpha_{0}(x)=0.5\left(0.02+\cos ^{2} x\right) x_{[0,1]}$;

(ii) $\alpha_{0}(x)=0.5\left(0.02+\sin ^{2} x\right) \chi_{[0,1]}$;

(iii) $\alpha_{0}(x)=\frac{\chi_{[0,1]}}{2} \frac{1+\exp \left[(x-0.5)^{2}\right]}{1+\exp \left[2(x-0.5)^{2}\right]}$;

where $\chi_{[0,1]}=1$ in $[0,1]$ and 0 otherwise. Here, $T=5, L=6, \Delta t=0.01$ and $\Delta x=0.02$, and the threshold is $\alpha_{\mathrm{thr}}=0.04(\operatorname{method} \mathrm{U})$ or $\alpha_{\mathrm{thr}}=$ 0.004 (method M). The second order method MUSCL significantly reduces the numerical diffusion (smoothening of numerical solutions at the points of discontinuity) [6]. The reduction of the numerical diffusion in method $\mathrm{M}$ compared to method $\mathrm{U}$ explains the reduction in the threshold value.

Figure 2 shows that the approximate solution obtained in the extended domain (scheme A) captures the properties of the analytical solution better than the one obtained in the scaled domain (scheme B), although it is less accurate towards the discontinuity at $\ell_{\mathrm{h}}^{\mathrm{N}}$ in method $\mathrm{U}$ owing to the high diffusion. But method $\mathrm{M}$ overcomes this disadvantage; the extended solution agrees well with the scaled solution towards the discontinuity, and remarkably better in the interior region. The recovered radius $\ell_{h}^{j}$ is in excellent agreement 


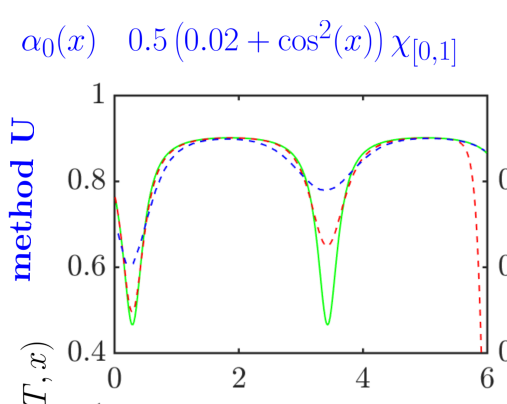

$$
0.5\left(0.02+\sin ^{2}(x)\right) \chi_{[0,1]}
$$

$\frac{\chi_{[0,1]}}{2} \frac{\left(1+\exp (x-0.5)^{2}\right)}{1+\exp (2(x-0.5))^{2}}$
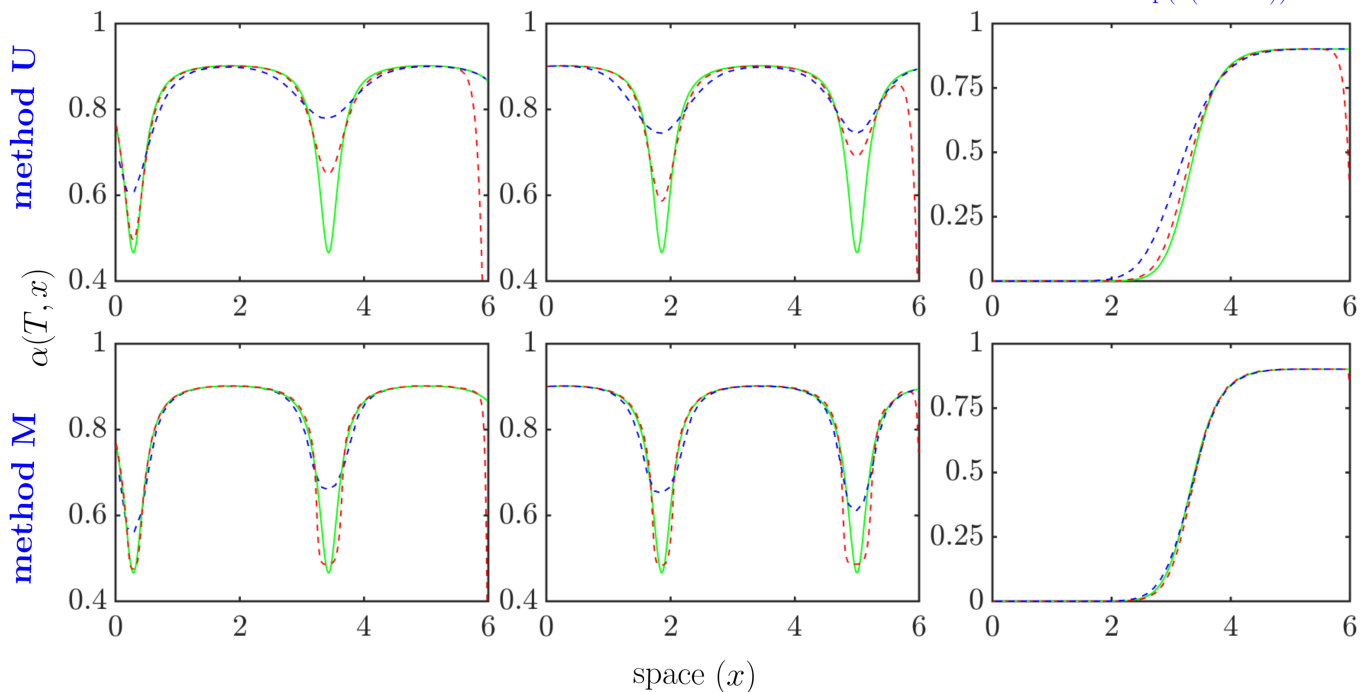

Figure 2: Numerical solutions for case 1 using (top) method U and (bottom) method $\mathrm{M}$ with initial data $\alpha_{0}(x)$ : (left) (i); (middle) (ii); and (right) (iii). In each plot, the red line is scheme A, the blue line is scheme B, and the green line is the analytical solution.

with the exact radius for both methods $\mathrm{M}$ and $\mathrm{U}$ with a suitable choice of threshold value (Tables 1 and 2).

We conclude this section by analysing the dependency of the recovered radius $\ell_{h}^{j}$ on the threshold value $\alpha_{\text {thr }}$ for the MUSCL method. The relative error $\Delta \ell_{h}=\left|\ell(T)-\ell_{h}^{N}\right| / \ell(T)$ at $T=5$ is used as a quantification of the error in the recovered radius. Two sets of experiments are conducted: (a) vary $\alpha_{\text {thr }}$ with a fixed $\Delta x$; (b) vary $\Delta x$ with a fixed $\alpha_{\text {thr }}$. Table 1 shows that there exists a wide range of $\alpha_{\mathrm{thr}}$ and $\Delta x$ for which the error remains below $1 \times 10^{-2}$. This low error assures the accuracy of the method while the selection of $\alpha_{\text {thr }}$ remains a pertinent problem. Table 2 shows that for method $U$ the ranges of $\alpha_{\text {thr }}$ and $\Delta x$ over which the error remains low are small, which is expected 
Table 1: $\Delta \ell_{h}$ for case 1 , method $M$.

\begin{tabular}{c|ccccc}
\multirow{2}{*}{$\Delta x$} & \multicolumn{5}{|c}{$\alpha_{\text {thr }}$} \\
\cline { 2 - 6 } & 0.010 & 0.008 & 0.006 & 0.004 & 0.002 \\
\hline 0.01 & $1.67 \times 10^{-3}$ & $1.67 \times 10^{-3}$ & $1.67 \times 10^{-3}$ & $1.67 \times 10^{-3}$ & $5.00 \times 10^{-3}$ \\
0.02 & $3.33 \times 10^{-3}$ & $3.33 \times 10^{-3}$ & $6.67 \times 10^{-3}$ & $1.33 \times 10^{-3}$ & $2.00 \times 10^{-3}$ \\
0.04 & $6.67 \times 10^{-3}$ & $6.67 \times 10^{-3}$ & $2.00 \times 10^{-2}$ & $2.67 \times 10^{-2}$ & $4.00 \times 10^{-2}$ \\
0.06 & $4.31 \times 10^{-2}$ & $1.58 \times 10^{-2}$ & $2.59 \times 10^{-2}$ & $4.60 \times 10^{-2}$ & $6.61 \times 10^{-2}$ \\
0.08 & $2.10 \times 10^{-2}$ & $7.66 \times 10^{-3}$ & $1.92 \times 10^{-2}$ & $3.26 \times 10^{-2}$ & $5.93 \times 10^{-2}$ \\
0.10 & $3.33 \times 10^{-2}$ & $1.67 \times 10^{-2}$ & $1.67 \times 10^{-2}$ & $5.00 \times 10^{-2}$ & $8.33 \times 10^{-2}$
\end{tabular}

Table 2: $\Delta \ell_{\mathrm{h}}$ for case 1 , method $\mathrm{U}$.

\begin{tabular}{c|cccc}
\multirow{2}{*}{$\Delta x$} & \multicolumn{4}{|c}{$\alpha_{\text {thr }}$} \\
\cline { 2 - 5 } & 0.04 & 0.03 & 0.02 & 0.01 \\
\hline 0.01 & $3.33 \times 10^{-3}$ & $3.33 \times 10^{-3}$ & $1.66 \times 10^{-2}$ & $3.83 \times 10^{-2}$ \\
0.02 & $3.33 \times 10^{-2}$ & $3.33 \times 10^{-3}$ & $1.33 \times 10^{-2}$ & $5.68 \times 10^{-2}$ \\
0.04 & $1.20 \times 10^{-1}$ & $7.33 \times 10^{-2}$ & $6.66 \times 10^{-3}$ & $6.00 \times 10^{-2}$ \\
\hline
\end{tabular}

considering the high numerical diffusion associated with this method.

\subsection{Case 2}

The two phase model with all the system variables treated as unknowns is now considered. The parameters are $\Delta \mathrm{t}=0.01, \Delta \mathrm{x}=0.01, \mathrm{~T}=228$ and $\mathrm{L}=25$, with $\alpha_{\mathrm{thr}}=0.004(\operatorname{method} \mathrm{M})$ and $\alpha_{\mathrm{thr}}=0.01$ (method $\left.\mathrm{U}\right)$ based on Tables 1 and 2. The initial condition is $\alpha_{0}(x)=0.8$ for $0 \leqslant x \leqslant 1$ and $\alpha_{0}(x)=0$ otherwise. Since the exact value of $\ell(T)$ is not available, the error is quantified as the relative difference between scheme $\mathrm{B}$ and scheme $\mathrm{A}$. The difference for method $\mathrm{U}$ is $6.18 \times 10^{-3}$ and for method $\mathrm{M}$ is $5.69 \times 10^{-3}$. Hence the moving boundary $\ell$ is well captured by both methods $U$ and $M$. Figure 3 shows that the numerical solution in the extended domain is in good agreement with the solution obtained from the scaled domain [2]. 

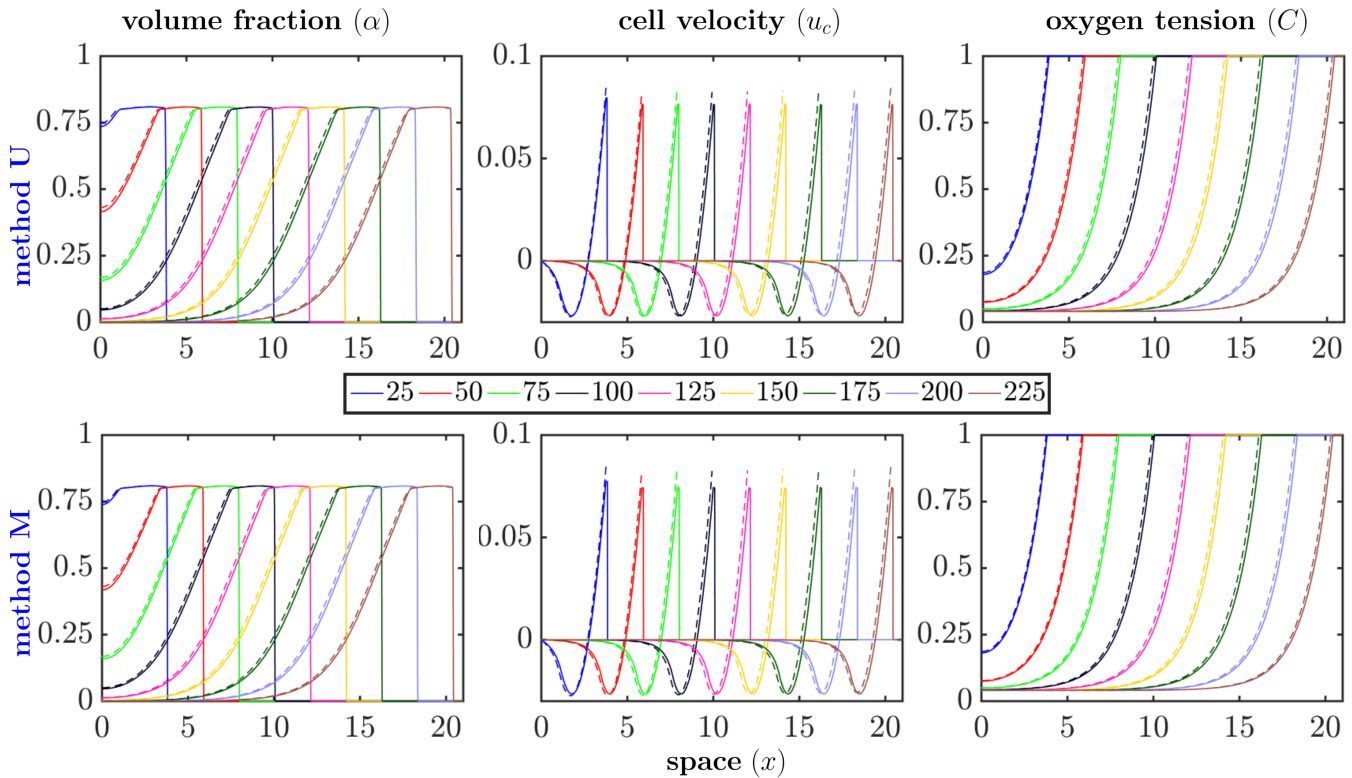

Figure 3: Numerical solutions for case 2 using (top) method U and (bottom) method M. The solid lines represent scheme A and the dotted lines represent scheme B. Each plot shows the variation of the corresponding variable: (left) $\alpha$; (middle) $\mathfrak{u}_{c}$; and (right) $\mathrm{C}$; with respect to space $\boldsymbol{x}$ at the fixed times $t=25,50, \ldots, 225$. The fixed times $t$ are distinguished by the coloured lines identified in the legend.

\section{Conclusion}

A novel numerical technique is developed to solve the two phase tumour growth problem and is tested against problems for which analytical solutions are known. For a fixed spatial mesh size the new method gives more accurate solutions than the standard method of solving in a scaled domain. The moving boundary is recovered from the numerical solution by comparing with a threshold value. It is found that an appreciable range of threshold values can be used along with higher order methods like MUSCL so that the 
error in the recovered radius can be kept low. The solutions obtained from this new technique show very good agreement with solutions obtained using standard methods. The reliability of this new method should be beneficial when extending the method to tumour growth problems in higher dimensions while not solving for the boundary explicitly.

Acknowledgement The author is grateful to A/Prof. Jérôme Droniou (Monash Univeristy), Dr. Jennifer Anne Flegg (University of Melbourne) and Prof. Neela Nataraj (I.I.T. Bombay) for the valuable suggestions and help.

\section{References}

[1] C. J. W. Breward, H. M. Byrne, and C. E. Lewis. "A multiphase model describing vascular tumour growth". In: B. Math. Biol. 65.4 (2003), pp. 609-640. DOI: 10.1016/S0092-8240 (03)00027-2 (cit. on p. C5).

[2] C. J. W. Breward, H. M. Byrne, and C. E. Lewis. "The role of cell-cell interactions in a two-phase model for avascular tumour growth". In: $J$. Math. Biol. 45.2 (2002), pp. 125-152. DOI: 10.1007/s002850200149 (cit. on pp. C2, C4, C9, C10, C12).

[3] H. M. Byrne, J. R. King, D. L. S. McElwain, and L. Preziosi. "A two-phase model of solid tumour growth". In: Appl. Math. Lett. 16.4 (2003), pp. 567-573. DOI: 10.1016/S0893-9659(03)00038-7 (cit. on p. C2).

[4] L. C. Evans. Partial Differential Equations. Vol. 19. Graduate Studies in Mathematics. American Mathematical Society, 1998. URL: https: //www.ams.org/publications/authors/books/postpub/gsm-19-R (cit. on p. C2). 
[5] R. Eymard, T. Gallouët, and R. Herbin. "Finite volume methods". In: Solution of Equation in $\mathbb{R}^{n}$ (Part 3), Techniques of Scientific Computing (Part 3). Vol. 7. Handbook of Numerical Analysis. Elsevier, 2000, pp. 713-1018. DOI: 10.1016/S1570-8659 (00)07005-8 (cit. on pp. C8, C9).

[6] B. van Leer. "Towards the ultimate conservative difference scheme. V. A second-order sequel to Godunov's method". In: J. Comput. Phys. 32.1 (1979), pp. 101-136. DOI: 10.1016/0021-9991(79)90145-1 (cit. on p. C10).

[7] J. Ward and J. R. King. "Mathematical modelling of avascular-tumor growth II: Modelling growth saturation". In: IMA J. Math. Appl. Med. 16 (1999), pp. 171-211. DOI: 10.1093/imammb16.2.171 (cit. on p. C5).

[8] J. Ward and J. R. King. "Mathematical modelling of avascular-tumour growth". In: Math. Med. Biol. 14.1 (1997), pp. 39-69. DOI: 10.1093/imammb/14.1.39 (cit. on p. C5).

\section{Author address}

1. Gopikrishnan C. Remesan, IITB-Monash Research Academy, IIT Bombay, Powai, Mumbai 400076, India mailto:gopikrishnan. chirappurathuremesan@monash.edu orcid:0000-0002-4507-4463 